\title{
Comprehensive Workspace Calibration for Visuo-Haptic Augmented Reality
}

\author{
Ulrich Eck, Frieder Pankratz, Christian Sandor, Gudrun Klinker, Hamid Laga
}

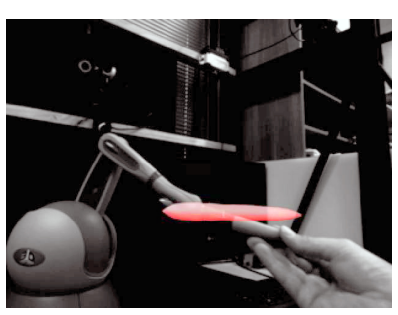

(a)

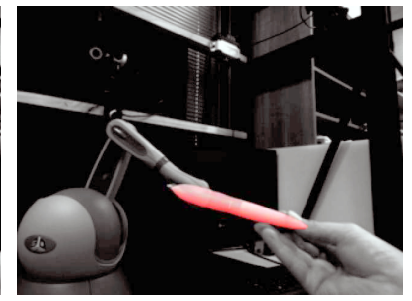

(b)

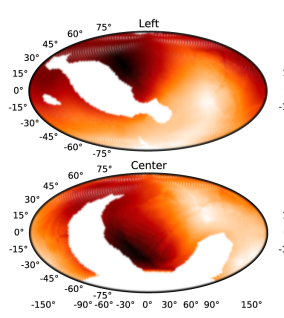

(c)

Figure 1: Visuo-haptic augmented reality system with a PHANToM haptic device. Virtual stylus overlaid (a) with only joint angle calibration, and (b) with our additional gimbal angle calibration. (c) Visualization of reduced orientation errors at four positions of the haptic workspace (in degrees on an unwrapped spherical heat-map with hammer projection).

\begin{abstract}
Visuo-haptic augmented reality systems enable users to see and touch digital information that is embedded in the real world. Precise co-location of computer graphics and the haptic stylus is necessary to provide a realistic user experience. PHANToM haptic devices are often used in such systems to provide haptic feedback. They consist of two interlinked joints, whose angles define the position of the haptic stylus and three sensors at the gimbal to sense its orientation. Previous work has focused on calibration procedures that align the haptic workspace within a global reference coordinate system and developing algorithms that compensate the non-linear position error, caused by inaccuracies in the joint angle sensors. In this paper, we present an improved workspace calibration that additionally compensates for errors in the gimbal sensors. This enables us to also align the orientation of the haptic stylus with high precision. To reduce the required time for calibration and to increase the sampling coverage, we utilize time-delay estimation to temporally align external sensor readings. This enables users to continuously move the haptic stylus during the calibration process, as opposed to commonly used point and hold processes. We conducted an evaluation of the calibration procedure for visuo-haptic augmented reality setups with two different PHANToMs and two different optical trackers. Our results show a significant improvement of orientation alignment for both setups over the previous state of the art calibration procedure. Improved position and orientation accuracy results in higher fidelity visual and haptic augmentations, which is crucial for fine-motor tasks in areas including medical training simulators, assembly planning tools, or rapid prototyping applications. A user friendly calibration procedure is essential for real-world applications of VHAR.
\end{abstract}

Index Terms: H.5.1. [Information Interfaces and Presentation]: Multimedia Information Systems-[Artificial, augmented and virtual realities]; H.5.2. [Information Interfaces and Presentation]: User Interfaces-[Haptic I/O ];

\section{INTRODUCTION}

Researchers have started to combine augmented reality (AR) and haptic interaction to enable users to see and touch digital informa-

IEEE International Symposium on Mixed and Augmented Reality 2014 Science and Technology Proceedings

10 - 12 September 2014, Munich, Germany

978-1-4799-6184-9/13/\$31.00 @2014 IEEE tion that is embedded in the real world. Such visuo-haptic augmented reality (VHAR) user interfaces with co-located visual augmentations and haptic interaction improve realism [2] and enable users to interact more precisely [6]. The haptic stylus is often augmented with some context-dependent tool like a drill for dental surgery training [17], a brush for virtual painting [18], or tools for rapid prototyping [5].

Precisely calibrating the components of a VHAR system (external trackers, cameras, haptic devices), and the spatial relations between them is essential to provide a realistic user experience. Specifically, the integration of haptic devices is not trivial. First, the haptic feedback needs to be precisely co-located with the visual augmentations. Second, the position and orientation of the haptic stylus need to be known in order to augment or to hide it [4].

Haptic devices for providing kinesthetic feedback are usually based on one of the two concepts: stylus- and grip-based devices for tool interaction and string-based systems for grasp tasks. Massie and Salisbury [13] developed the widely used stylus-based PHANToM haptic device, which consists of two interlinked joints. The angles of these joints define the position of the haptic stylus, commonly called haptic interface point (HIP). Three sensors at the gimbal sense the stylus orientation.

Various solutions have been proposed to integrate haptic devices into AR environments [21, 11,7], but all of them focused on the calibration of the HIP position, which is very important to co-locate linear force feedback. However, the stylus orientation also needs to be calibrated to enable precise augmentations (see Figure 1a and 1b). In this paper we present a comprehensive workspace calibration procedure for VHAR, which reduces position and orientation errors of the haptic stylus significantly.

Contribution. Our main contribution is an algorithm for calibrating the three gimbal angles, which results in a reduction of the average orientation error of more than $40 \%$. Corrected orientations of the haptic stylus are required for precise overlays of virtual tools (see Figure 1a and 1b) and accurate co-location of haptic feedback. Furthermore, we present an improved calibration procedure, utilizing time-delay estimation to temporally align external sensor readings, resulting in reduced time required, increased sampling coverage, and a decreased average position error of $20 \%$. Improved position and orientation accuracy results in higher fidelity visual and haptic augmentations, which is crucial for fine-motor tasks in areas including medical training simulators, assembly planning tools, or rapid prototyping applications. A user friendly calibration proce- 
dure is essential for real-world applications of VHAR.

Our calibration procedure is as follows: First, we use tooltip calibration to estimate the HIP position in tracker coordinates and absolute orientation calibration to align the haptic workspace with the tracker. Second, we determine the time-delay between the external tracker and the haptic device in order to compensate it. Subsequent steps in the procedure will receive temporally aligned measurements. Third, we perform a workspace calibration based on Harders' method [7] to calibrate the joint angles. In order to further improve the position accuracy, we repeat absolute orientation and joint angle calibration.

Finally, we calibrate the three gimbal angles. First we need to determine the physical orientation of the haptic stylus in relation to the attached tracking target as a reference. Next, we calibrate the first two gimbal angles by minimizing the angle between the longitudinal axis of the haptic stylus and the reference. Finally we compensate the error of the third sensor-the rotation around longitudinal axis-to complete the calibration.

We evaluated our calibration method in a low-fidelity setup $(L F)$ consisting of a PHANToM Omni and an OptiTrack IR tracker and a high-fidelity setup $(H F)$ with a PHANToM Premium $1.56 \mathrm{DOF}$ and an A.R.T IR Tracker. Our results show that calibrating gimbal sensors reduces the average orientation error by $44 \%$ for $L F$ and $42 \%$ for $H F$ in comparison uncalibrated setups. We also show that the average position accuracy can be improved by $22 \%$ for $L F$ and $19 \%$ for $H F$ in comparison to Harder's method.

The rest of this paper is structured as follows: in Section 2, we highlight the novelty of our workspace calibration by comparing it against related work. In Section 3, we describe our calibration procedure and show how it differs from Harders' method. In Section 4, we discuss the algorithm used for calibrating the gimbal angles. In Section 5, we present the evaluation that we performed. Finally, Section 6 concludes by discussing the generalizability of our results and presenting future work.

\section{Related Work}

Previous research has investigated the integration of haptic devices in AR systems. A key requirement is the precise co-location of the haptic stylus with the visual augmentations to provide realistic force feedback and to enable precise visual overlays.

Vallino and Brown [21] pioneered the co-location of a PHANToM Omni haptic device in AR by determining an affine transformation between the haptic workspace and a world coordinate reference. They used four non-planar points, which are captured in both coordinate systems to estimate the affine transformation. Their method does not take into account the fact that the poses reported by the haptic device are distorted, due to errors in the joint angle sensors. Their method works only for static camera poses. Ikits et al. [11] presented a calibration method for haptic devices using a planar grid to correct the haptic stylus position. Their method significantly improves position accuracy in the region where the calibration grid was placed; However, in other areas of the workspace this method fails to effectively compensate distortions. Wang et al. [22] derived a mathematical model of the co-location error for VHAR systems with half-mirror displays and defined error metrics. They propose a calibration method using their model and evaluate it using a precise mechanical tracker. Their approach results in improved co-location for half-mirror display setups, but ignores colocation errors caused by sensors in haptic devices.

The most important previous research is the work by Harders et al. [7]. They presented a calibration procedure for VHAR workspaces and an algorithm to compensate the non-linear distortion of position measurements of PHANToM haptic devices for the complete workspace. They used an external tracker and rigidly attached a tracking target to the haptic stylus to perform an openloop calibration [8]. Their procedure is as follows: First, they de- termine the position of the HIP in relation to the tracking target using tooltip calibration as a reference. Second, they collect position measurements reported from the haptic device and the corresponding tip positions from the tracker to estimate a 6DOF transform between the haptic workspace and the external tracker. Finally, they sample more point correspondences covering the complete haptic workspace in order to compensate the joint angle sensor errors, which are causing the position errors on the workspace boundaries. They model the sensor errors using a linear system and use Levenberg-Marquardt optimization to minimize the squareddistance between reference positions and positions calculated using the forward kinematic model of the PHANToM from Cavusoglu et al. [3].

Knörlein et al. [12] compared tracker-based calibration with an alternative approach using a physical calibration object with three perpendicular planes. By constraining the measured hip positions to these planes, they can effectively compensate position errors caused by the joint angle sensors. This approach cannot be easily adapted to orientation calibration.

Huber et al. [16] presented Ubitrack, an ubiquitous tracking and sensor fusion framework, which forms the basis of our implementation. Spatial relationship patterns [15] are used to define the spatial relations between entities in a system. Ubitrack implements an asynchronous dataflow network and provides components for calibration, persistence, and device drivers. Ubitrack also facilitates modeling of concatenated transforms and sensor fusion, interpolation and filtering. This allows to exchange the PHANToM device and the external tracker with other devices without the need to write a single line of code, while using the same calibration pipeline.

Our setup consists of two unsynchronized sensor inputs: a haptic device and an external tracker. Since we want to avoid modifications to the sensors in order to achieve hardware synchronization, we use a software-based approach. We determine the timedelay between our sensors using an algorithm presented by Huber et al. [10]. With a known delay, the Ubitrack pipeline can then temporally align the measurements. The basic idea in [10] is to gather measurements with accurate timestamps from two different sensors that have been transformed into a common coordinate system. Using segments of these measurement streams, the similarity between to two signals is computed, while one of the slices is shifted in time. The shift in time, which maximizes the similarity between the signals, corresponds to the time-delay between the sensors. The temporal alignment of the measurements enables a continuous collection of samples for increased workspace coverage and faster calibration.

Our work extends Harders and colleagues' method to all six device sensors, resulting in a precise 6DOF alignment of the haptic stylus and visual overlays (see Figures $1 \mathrm{a}, \mathrm{b}$, and Section 4). We additionally propose an improved calibration procedure that consists of time-delay compensation and the repetition of workspace alignment and joint angle calibration, which is discussed next.

\section{Calibration Procedure}

In this section, we provide details about our improved calibration procedure and highlight the differences to Harders' method. A schematic overview of our VHAR workspace setup is given in Figure 2. An external IR tracker $(E T)$ is used to track a camera with an attached target $\left(C_{\text {target }}\right)$. A second tracking target $\left(H I P_{\text {target }}\right)$ is attached to the stylus of the haptic device $(H D)$ as reference for calibration. The spatial relation between the haptic workspace $\left(H D_{\text {origin }}\right)$ and the external tracker $\left(E T_{\text {origin }}\right)$ is static.

Initially, we calibrate the external tracker using the vendorsupplied tool, determine the camera intrinsics, and estimate the 6DOF transform between the $C_{\text {target }}$ and the camera coordinate system $(C)$ using the hand-eye calibration method by Tsai and Lenz [19]. We initialize the PHANToM haptic device using the vendor- 


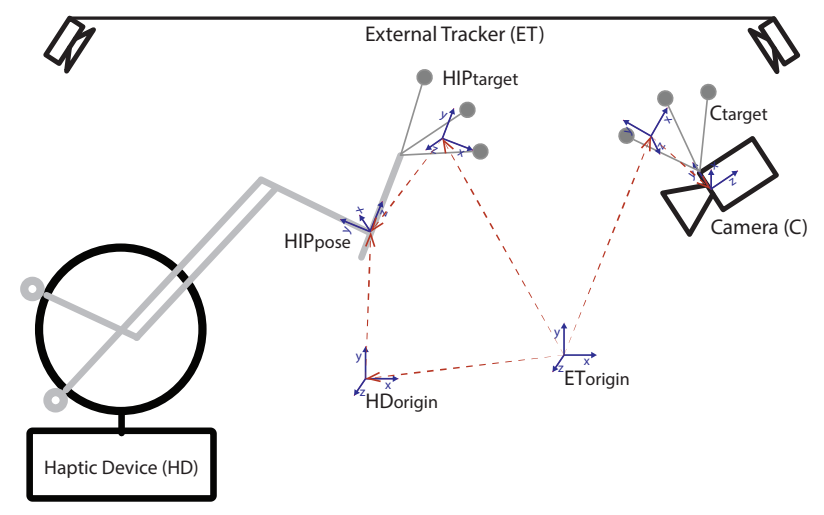

Figure 2: Our setup for for VHAR workspace calibration and its spatial relations.

supplied tool and rigidly attach a tracking target to its stylus. The PHANToM Omni provides an inkwell for automatic calibration of the device in a fixed physical location. However, PHANToM Premium devices have no inkwell for calibration, which results in unpredictable reset positions.

Table 1: Overview of the calibration procedure

\begin{tabular}{lll}
\hline Step & Description & Relation \\
\hline Time Delay Estimation & Tooltip [20] & $H I P_{\text {target }}-H I P_{\text {pose }}$ \\
Section 3.1 & Absolute Orientation [9] & $E T_{\text {origin }}-H D_{\text {origin }}$ \\
& Time Delay Estimation [10] & $\Delta t(H D-E T)$ \\
\hline Position Calibration & Joint-Angle Calibration [7] & $H I P_{\text {pose }}$ \\
Section 3.2 & Absolute Orientation [9] & $E T_{\text {origin }}-H D_{\text {origin }}$ \\
& Joint-Angle Calibration [7] & $H I P_{\text {pose }}$ \\
\hline Orientation Calibration & Orientation Reference & $H I P_{\text {target }}-H I P_{\text {pose }}$ \\
Section 4 & Gimbal-Angle Calibration & $H I P_{\text {pose }}$ \\
\hline
\end{tabular}

An overview of the workspace calibration procedure is given in Table 1 . The procedure starts by determining the time-delay between $E T$ and $H D$ (Section 3.1). Then we use Harders' method to calibrate the joint angles, and repeat absolute orientation and joint angle calibration to reduce position errors (Section 3.2). Finally we calibrate the gimbal angles, see Section 4.

\subsection{Time Delay Estimation}

The time-delay estimation algorithm expects two unsynchronized streams of corresponding 3D positions in a common coordinate system, $H D_{\text {origin }}$ in our case. Therefore we need to estimate the transform between the external tracker $E T_{\text {origin }}$ and haptic device $H D_{\text {origin. }}$ This is done in two steps: First, we estimate the $H I P_{\text {pose }}$ position in tracker coordinates $H I P_{\text {target }}$ using the tooltip calibration method presented by Tuceryan et al. [20]. We prefer a mechanical stand over a fixation force as proposed by Harders for increased accuracy. Second, we determine a 6DOF transform between $E T_{\text {origin }}$ and $H D_{\text {origin }}$ using the absolute orientation algorithm presented by Horn [9].

In the next step, the stylus is moved on an arbitrary path with non-constant distance to the workspace origin. The corresponding position measurements from $H I P_{\text {target }}$ and the haptic stylus are then reduced in dimensionality using the Euclidean norm (in this case the distance from the workspace origin). The resulting signals are then correlated. In [10] they note that the registration of the two tracking systems does not have to be very precise, since the time-delay estimation is rather robust against spatial registration errors. Thus we can use the same registration procedure used in subsequent steps of the calibration, where the time-delay is not yet compensated. In our setup, the time-delay estimation results in a delay of $9 \mathrm{~ms}$ for the PHANToM Omni setup (the measurements

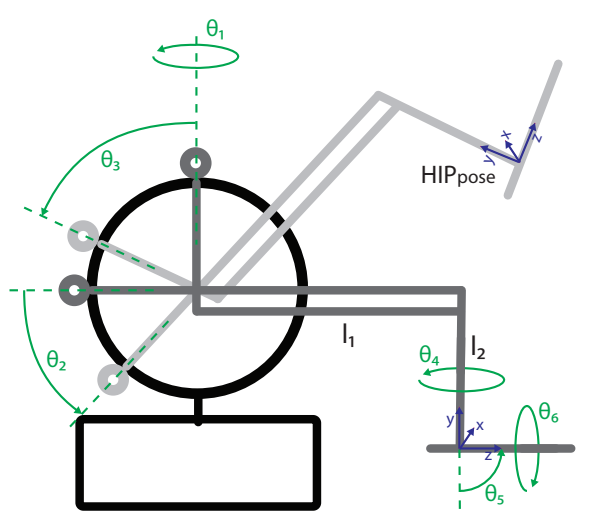

Figure 3: 6DOF forward kinematic model for a PHANToM haptic device.

of the Naturalpoint OptiTrack system arrive 9ms after the measurements from the PHANToM Omni on the host computer) and $19 \mathrm{~ms}$ for the PHANToM Premium 1.5 setup.

Different strategies like buffering, linear interpolation, or Kalman filters can be applied to temporally align the measurements. Since the update rate of PHANToM devices is very high $(1 \mathrm{KHz})$ compared to the reference tracking systems $(60-100 \mathrm{~Hz})$, we use a simple buffer mechanism that provides us the temporally closest PHANToM measurement for a measurement from the reference tracking system. All subsequent steps will now receive temporally aligned measurements.

\subsection{Position Calibration}

Next we compensate the HIP position errors using Harders' method. Since all measurements are temporally aligned, operators can freely move the stylus to sample point correspondences for the Levenberg-Marquardt optimization. In order to further simplify the data collection, we automatically trigger the sampling when the stylus has travelled a predefined distance, which is varied between $1-10 \mathrm{~cm}$ depending on the haptic workspace volume. To this end, we have reduced the average position error from $5.89 \pm 2.39 \mathrm{~mm}$ to $2.3 \pm 0.84 \mathrm{~mm}$ for a PHANToM Omni and from $5.99 \pm 1.74 \mathrm{~mm}$ to $3.14 \pm 0.7 \mathrm{~mm}$ for a PHANToM Premium 1.5.

Since the absolute orientation calibration step initially uses uncalibrated positions from the haptic device, we capture only point correspondences in the center of the haptic workspace, which can lead to orientation errors in the transform. In order to improve the alignment of the two coordinate systems, we now repeat the absolute orientation step with samples covering a larger volume and to further improve the joint angle calibration we repeat it as well. This results in reduced average position errors for the PHANToM Omni setup of $1.8 \pm 0.33 \mathrm{~mm}$ and $2.55 \pm 0.71 \mathrm{~mm}$ for a PHANToM Premium 1.5.

\section{Orientation Calibration}

The calibration of gimbal angles requires a known reference that can be used to determine the correction parameters for the sensor readings by minimizing the orientation error between the reference and the calculated stylus pose. Such a reference that represents the physical orientation of the haptic stylus can be determined in two ways: using a custom, precisely manufactured, and calibrated tracking target with well-known properties, or by exploiting the mechanical and geometrical properties of the haptic stylus with attached tracking target. The first approach is expensive and not easily adaptable to new combinations of tracking systems and haptic devices. Therefore, we chose the latter, more general approach. It is comparable to the tooltip calibration step, as it finds the reference orientation in relation to some arbitrarily attached tracking target. In this section, we present the algorithm to determine the 


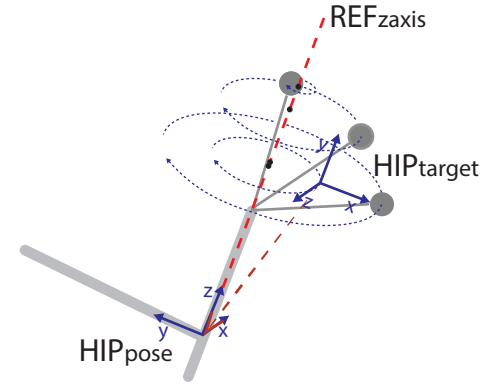

(a)

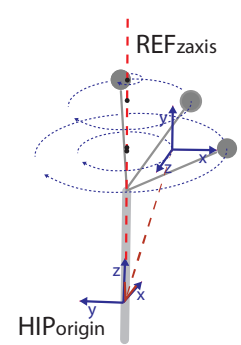

(b)
Figure 4: $H I P_{\text {target }}$ origin and its markers travel on a circular path around the physical z-axis of the haptic stylus when it is rotated around its longitudinal axis. The centers of the circles, which are marked by black dots, are on a straight line through the $H I P_{\text {pose }}$ position. (a) stylus rotation in tracker coordinates. (b) stylus rotation in HIP $P_{\text {origin }}$ coordinates.

reference orientation, then we show how the reference orientation can be used to calibrate the gimbal angles.

As a prerequisite for our approach, it is essential to process only temporally aligned pose correspondences from the haptic device and the external tracker. By comparing the poses reported from the haptic device with the physical pose in a high-speed video $(120 \mathrm{~Hz})$, we found, that the gimbal sensor readings are delayed. Geomagic support acknowledged this undocumented behavior and stated that the gimbal measurements are by default interpolated over 180 frames to avoid jitter. This interpolation leads to delayed changes in orientation and therefore incorrect pose measurements. The interpolation can be configured only by editing the parameter FilterGimbalSize in the device configuration file. We have set this parameter to a value of 0 to avoid any delay in the reported orientation.

Furthermore, the forward kinematics equations from Cavusoglu et al. [3] need to be extended to include the gimbal angles in order to compute a full 6DOF HIP Pose (see Figure 3 ). We report all equations in right-handed coordinates. Shortcuts are provided for $\sin ()=.s($.$) and \cos ()=.c($.$) .$

Let $l=\left(l_{1}, l_{2}\right)$ be the vector of joint lengths and $\theta_{j a}=\left(\theta_{1}, \theta_{2}, \theta_{3}\right)$ the vector of joint angles of the haptic device. The translation from the haptic workspace origin to the HIP position $T_{H I P}$ is given by:

$$
T_{H I P}\left(\theta_{j a}, l\right)=\left[\begin{array}{c}
-s\left(\theta_{1}\right)\left(c\left(\theta_{2}\right) l_{1}+l_{2} s\left(\theta_{3}\right)\right) \\
-c\left(\theta_{3}\right) l_{2}+l_{1} s\left(\theta_{2}\right)+l_{2} \\
\left.c\left(\theta_{1}\right)\left(c \theta_{2}\right) l_{1}+l_{2} s\left(\theta_{3}\right)\right)-l_{1}
\end{array}\right]
$$

and the orientation of the second arm by:

$$
R_{123}\left(\theta_{j a}\right)=\left[\begin{array}{ccc}
c\left(\theta_{1}\right) & s\left(\theta_{1}\right) s\left(\theta_{3}\right) & -s\left(\theta_{1}\right) c\left(O_{3}\right) \\
0 & c\left(\theta_{3}\right) & s\left(\theta_{3}\right) \\
s\left(\theta_{1}\right) & -s\left(\theta_{3}\right) c\left(\theta_{1}\right) & c\left(\theta_{1}\right) c\left(\theta_{3}\right)
\end{array}\right]
$$

As detailed in Figure 3 the rotations $\theta_{g a}=\left(\theta_{4}, \theta_{5}, \theta_{6}\right)$ measured by the gimbal sensors can be expressed as:

$$
R_{4}=\left(\left[\begin{array}{l}
0 \\
1 \\
0
\end{array}\right], \theta_{4}\right) R_{5}=\left(\left[\begin{array}{l}
1 \\
0 \\
0
\end{array}\right],-\theta_{5}\right) R_{6}=\left(\left[\begin{array}{l}
0 \\
0 \\
1
\end{array}\right],-\theta_{6}\right)
$$

where $(v, \theta)$ refers to a rotation around axis $v$ with an angle $\theta$. The rotations are concatenated, which results in a complete rotation $R_{H I P}$ :

$$
R_{H I P}\left(\theta_{j a}, \theta_{g a}\right)=R_{123} R_{4} R_{5} R_{6}
$$

and a full $6 \mathrm{DOF}$ pose for the haptic stylus $H I P_{\text {pose }}$ :

$$
H I P_{\text {pose }}\left(\theta_{j a}, \theta_{g a}, l\right)=\left[\begin{array}{cccc} 
& R_{H I P} & & T_{H I P} \\
0 & 0 & 0 & 1
\end{array}\right]
$$
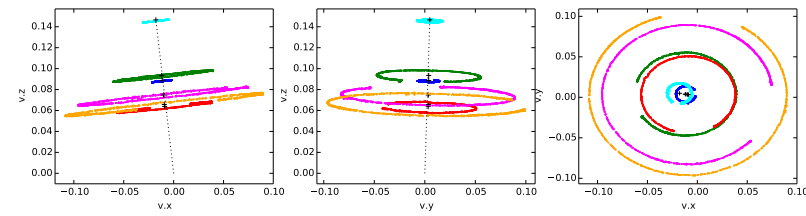

Figure 5: $R E F_{\text {zaxis }}$ is determined by finding centers of circles described by markers and fitting a line through the stylus origin.

\subsection{Reference Orientation}

The physical orientation of the haptic stylus is initially unknown. We present a novel approach to determine the stylus orientation in relation to $H I P_{\text {target }}$. When the stylus is rotated around its longitudinal axis, the attached tracking target and its markers travel on a circular path around the z-axis (see Figure $4 a$ ). The centers of these circles define a straight line $R E F_{\text {zaxis }}$ through the HIP and represent the physical orientation of the stylus. Aligning the z-axis of $H I P_{\text {pose }}$ with $R E F_{\text {zaxis }}$ during the optimization yields correction factors for $\theta_{4}$ and $\theta_{5}$. Another property of the haptic stylus is, that the rotation around its longitudinal axis is mechanically limited in both directions. We use this property to calibrate $\theta_{6}$.

When users rotate the stylus around $R E F_{\text {zaxis }}$ without any mechanical fixture, it is impossible to avoid a change in orientation on the other axes. Therefore, it is not possible to simply fit the circles on raw measurements. Since we have corresponding sensor readings from joint and gimbal sensors of the haptic device, we can compensate this movement using the forward kinematic pose of $\theta_{1}$ to $\theta_{5}$

$$
H_{I} P^{\prime}\left(\theta_{j a}, \theta_{g a}, l\right)=\left[\begin{array}{cccc} 
& R_{123} R_{4} R_{5} & & T_{H I P} \\
0 & 0 & 0 & 1
\end{array}\right]
$$

Let $v$ be the vector of $H I P_{\text {target }}$ marker positions, $P$ the $H I P_{\text {target }}$ pose, $F$ the corresponding pose calculated using $H I P^{\prime}$, and $X$ the transform between $E T_{\text {origin }}$ and $H D_{\text {origin }}$. As shown in Figure $4 \mathrm{~b}$, each $v$ is transformed into stylus coordinates $H I P_{\text {origin }}$ using

$$
v_{i}^{\prime}=F_{i}^{-1}\left(X v_{i}\right)
$$

As shown in Figure 5, circles can now be fitted in the $x-y$ plane using orthogonal distance regression [1]. This results in a series of $3 \mathrm{D}$ points defining $R E F_{\text {zaxis }} z$ in stylus coordinates, which is calculated using singular value decomposition. To obtain $z_{\text {ref }}$ in $E T_{\text {origin }}$ coordinates we transform $z$ using $n$ correspondences of $P$ and $F$

$$
z_{\text {ref }}=\frac{1}{n} \sum_{i=1}^{n}\left(X P_{i}\right)^{-1}\left(F_{i} z\right)
$$

At this stage, we obtained one reference axis, which allows us to minimize errors for $\theta_{4}$ and $\theta_{5}$ (see Section 4.2). Next, we determine the reference needed for calibrating $\theta_{6}$.

The stylus rotation around its z-axis is mechanically limited. We advise users to rotate the stylus towards both extremes. This mechanical limit is then also reflected in the circular paths described by the HIP target markers as shown in Figure 5. Each path has a gap that corresponds to the mechanical limit of the rotation. The opposite side of the center of the gap defines the origin of $\theta_{6}$, while the upper and lower bounds define its range. We use this correspondence to calibrate $\theta_{6}$.

Let $C$ be the vector of $2 \mathrm{D}$ positions in the $\mathrm{x}-\mathrm{y}$ plane of the circle with the largest value for radius $r$ divided by its residual. We transform $C$ into a unit circle $C^{\prime}=\frac{C}{r}$ and compute the vector $\alpha$ consisting of angles $\alpha_{i}$ that correspond to each $C_{i}^{\prime}$. The lower bound $\alpha_{l}$ and the upper bound $\alpha_{u}$ of the gap in $C^{\prime}$ is determined using a histogram with 360 bins. The origin reference for $\theta_{6}$ is $\alpha_{o}=\frac{\alpha_{u}-\alpha_{l}}{2}+\pi$. As a final step, we compute the reference vector for calibration as $\hat{\alpha}=-\alpha+\alpha_{o}$.

In the next section, we discuss the error minimization for gimbal angles using $z_{\text {ref }}$ and $\hat{\alpha}$. 

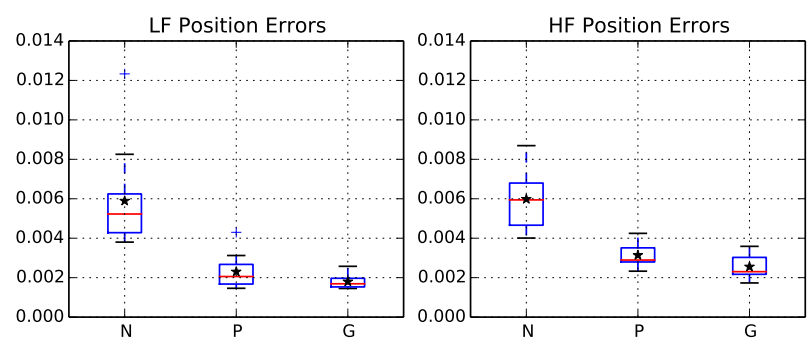

Figure 6: Position errors as euclidean distance in $\mathrm{mm}$ for two setups and three conditions. (N: no calibration, P: Harders' method, G: our method)

\subsection{Gimbal Sensor Error Compensation}

We consider two models for the deviation between gimbal angles and sensor measurements. First, a linear function $\theta_{g a}=k_{g a} \psi_{g a}+$ $m_{g a}$ where $\psi_{g a}$ represents the vector of measured gimbal angles, $k_{g a}$ the gains, and $m_{g a}$ the the offsets. Second, we model the gimbal angle errors using a quadratic function $\theta_{g a}=j_{g a} \psi_{g a}^{2}+k_{g a} \psi_{g a}+m_{g a}$ with an additional factor $j_{g a}$ for the squared angle. We evaluated both error models, because gimbal sensors in a PHANToM are potentiometers as opposed to digital encoders for the joint angles.

Let $\phi_{j a}=\left(k_{j a}, m_{j a}\right)$ be the correction factors for $\psi_{j a}$, which have been determined during the position calibration. Let $\phi_{g a}=$ $\left(j_{g a}, k_{g a}, m_{g a}\right)$ be the unknown correction factors for $\psi_{g a}$, setting $j_{g a}=0$ for the linear error model. The corrected stylus rotation can be expressed as $R^{\prime}\left(\psi_{j a}, \phi_{j a}, \psi_{g a}, \phi_{g a}\right)$ by substituting $\phi_{j a}$ and $\phi_{g a}$ into (4).

First we obtain the correction factors $\phi_{45}$ for $\theta_{4}$ and $\theta_{5}$ by performing a nonlinear optimization using the Levenberg-Marquardt algorithm. This minimizes the angle between the $\mathrm{z}$-axis of $H I P_{\text {pose }}$ and $\hat{z}=R_{\text {target }} \cdot z_{\text {ref }}$.

$$
\min _{\phi_{45}} \sum_{i=1}^{N} 1-\hat{z}_{i} \cdot\left(R_{i}^{\prime}\left(\psi_{j a}, \phi_{j a}, \psi_{g a}, \phi_{45}\right) \cdot\left[\begin{array}{l}
0 \\
0 \\
1
\end{array}\right]\right)
$$

Determining the correction factors $\phi_{6}$ for $\theta_{6}$ is straightforward, since we have obtained direct correspondences in $\hat{\alpha}$. Let vector $\beta$ be the vector of $\theta_{6}$ measurements. We minimize the error using least-squares optimization

$$
\min _{\phi_{6}} \sum_{i=1}^{N}\left(j_{6} \beta_{i}^{2}+k_{6} \beta_{i}+m_{6}\right)-\hat{\alpha}_{i}
$$

The final correction factors $\phi_{g a}$ are then composed from $\phi_{45}$ and $\phi_{6}$.

\section{Evaluation}

We evaluated our workspace calibration method in a low-fidelity $(L F)$ and a high-fidelity $(H F)$ setup in order to verify its correctness. In this section we present the numerical evaluation of repeated calibrations for our setups and discuss the results.

The $L F$ setup covers a small workspace and consists of a PHANToM Omni, a Naturalpoint OptiTrack IR tracking system, and a PointGrey Flea2 firewire camera using a single workstation (Intel Core I7 3.5GHz, 16GB RAM, NVidia GForce GTX 570). The $H F$ setup is a room-size installation with a PHANToM Premium 1.5 6DOF, an A.R.T ARTTRACK IR tracking system connected via $1 \mathrm{~GB} / \mathrm{s}$ network and a IDS uEye usb camera on a workstation (Intel Core I5 3.4GHz, 8GB RAM, NVidia GForce GTX 460).

We have conducted a series of calibration sessions for each setup: twelve sessions for $L F$ and six for $H F$. During each session we recorded all sensor data for evaluation as well as the calibration results. Recordings consist of triggered samples used for error minimization as well as raw sensor data-streams. All errors are calculated based on raw sensor data using the correction parameters for joint and gimbal angles within each session.
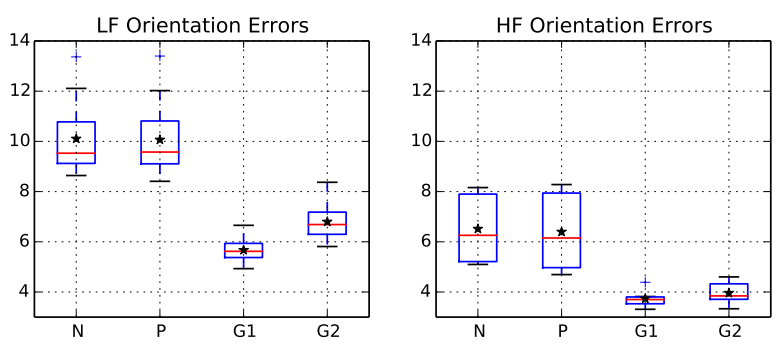

Figure 7: Orientation errors in degrees for two setups and four conditions ( $N$ : no calibration, $\mathrm{P}$ : joint angle calibration only, G1: joint and gimbal angle calibration with linear error model, G2: joint and gimbal angle calibration with quadratic error model)

We report the position error as Euclidean distance between the reference $H I P_{\text {target }}$ and the calculated $H I P_{\text {pose }}$ for three conditions in both setups: $N$ no calibration, $P$ joint angle calibration using Harders' method, and $G$ repeated joint angle calibration as presented in Section 3. The resulting position errors are shown in Figure 6.

In $L F$ the average position error for $N$ is $5.89 \pm 2.39 \mathrm{~mm}$, improved in $P$ to $2.3 \pm 0.84 \mathrm{~mm}$, and most accurate in $G$ with $1.8 \pm$ $0.33 \mathrm{~mm}$. In $H F$ the average position error for $N$ is $5.99 \pm 1.74 \mathrm{~mm}$, improved in $P$ to $3.14 \pm 0.7 \mathrm{~mm}$, and most accurate in $G$ with $2.55 \pm 0.71 \mathrm{~mm}$.

We report the orientation error for $\theta_{4}$ and $\theta_{5}$ in degrees between the estimated $R E F_{\text {zaxis }}$ and the $\mathrm{z}$-axis of the calculated $H I P_{\text {pose }}$ for four conditions in both setups: $N$ no calibration, $P$ joint angle calibration using Harders' method, $G 1$ gimbal angle calibration with linear error model, and $G 2$ gimbal angle calibration with quadratic error model. The resulting orientation errors are shown in Figure 7.

In $L F$ the average orientation error for $N$ is $10.11 \pm 1.43^{\circ}$, similar in $P$ to $10.07 \pm 1.47^{\circ}$, improved in $G 2$ with $6.8 \pm 0.76^{\circ}$, and most accurate in $G 1$ with $5.67 \pm 0.51^{\circ}$. In $H F$ the average orientation error for $N$ is $6.51 \pm 1.5^{\circ}$, similar in $P$ to $6.4 \pm 1.7^{\circ}$, improved in $G 2$ to $3.96 \pm 0.49^{\circ}$, and most accurate in $G 1$ with $3.74 \pm 0.37^{\circ}$.

Figure 8 illustrates the distribution of the orientation error caused by $\theta_{4}$ and $\theta_{5}$ for a typical calibration session in $L F$. Measurements were recorded with a fixed stylus position close to the workspace origin. The error in spherical coordinates $\left(H D_{\text {origin }}\right)$ is visualized using a heat-map that is projected using a hammer projection. White areas contain no measurements due to mechanical limits of the haptic stylus; higher values represent a larger error.

Figure 1c visualizes the orientation calibration results within the workspace. Four heat-maps show the reduction of orientation errors in degrees at four positions of the haptic workspace using an unwrapped spherical heat-map with hammer projection; higher values represent more error compensation.

The results for gimbal calibration suggest that a linear error model is better suited than the quadratic model. This contradicts our initial hypothesis that errors of a potentiometer are typically non-linear, as stated by Mooring et al. [14]. We plan to further investigate this topic in future work.

We have shown that the gimbal calibration significantly improves the accuracy of the reported stylus orientation, which enables precise overlays and improves torque feedback co-location. Our results also indicate that time-delay estimation can be used to temporally align sensor input from unsynchronized sources, which streamlines the data collection process and can improve workspace coverage.

\section{Conclusions AND FUtURE WORK}

Our comprehensive workspace calibration method enables accurate visual overlays on a haptic stylus and precise co-location of haptic torque feedback in VHAR systems. We presented a novel approach to estimate the physical orientation of a haptic stylus; this enables us 


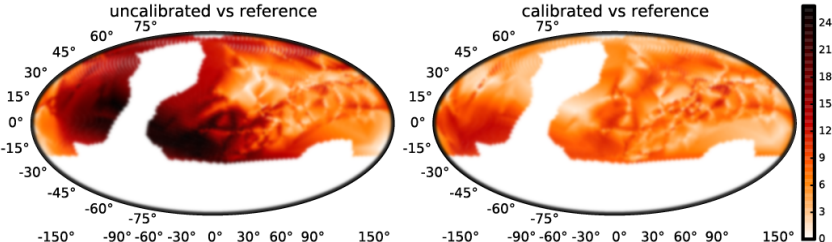

Figure 8: Angle error in degrees heat-map before and after the calibration measured near the workspace center and displayed in spherical coordinates using hammer projection; white areas contain no measurements.

to compensate errors in the gimbal sensors, improving orientation accuracy by more than $40 \%$. Additionally, the integration of timedelay estimation enables users to calibrate their workspace faster and more conveniently. Our calibration procedure further improves the position accuracy by $20 \%$.

The algorithm for gimbal angle calibration is generic and can be used for any pen-based haptic device with similar mechanical properties. The integration of time-delay estimation is also applicable to many systems, which integrate multiple, unsynchronized sensor inputs.

We believe that the quality of co-location can be further improved. The most important input to our algorithm is the pose provided by the IR tracking system, whose accuracy depends heavily on the tracking target. The tracking target can only be mounted at a certain distance from the HIP due to mechanical constraints. This leads to increased errors in the reference position, which are caused by orientation errors in the $6 \mathrm{D}$ pose of the tracking target. A larger tracking target could reduce the orientation error, but would then limit the reachable poses during the calibration process. In a follow up evaluation, we plan to use a precise mechanical tracker in order to determine the error caused by external IR trackers.

Besides improving the co-location accuracy, we also plan to further improve the overall calibration process. First, we expect to be able to reduce the required time by reusing data from previous steps. Second, we plan to simplify the process by adding visual and haptic guides, so that non-expert users can perform a workspace calibration.

In order to further reduce the required time for calibration, we plan to eliminate as many manual steps in the process as possible. Obviously, the repeated steps for absolute orientation and joint angle calibration can be skipped if data from previous session is reused. Tooltip calibration and gimbal angle calibration require the same input as well. In order to reuse data for multiple calibration steps, it is necessary to analyze the recorded sensor data streams and select best fitting samples for the current task.

The current calibration process requires too much knowledge about the steps and offers no guidance to users. Visual and haptic cues have not been utilized, although they are readily available. In future work, we plan to implement and evaluate haptic and visual cues that guide non-expert users during the calibration process.

Improved position and orientation accuracy results in higher fidelity visual and haptic augmentations, which is crucial for finemotor tasks in areas including medical training simulators, assembly planning tools, or rapid prototyping applications. Our work is an important step towards a complete and user friendly workspace calibration, which is essential for enabling real-world VHAR applications.

\section{ACKNOWLEDGEMENTS}

We greatly acknowledge the support from our colleagues at Technische Universität München: Pascal Fallavollita from NARVIS for providing access to their PHANToM Premium 1.5 6DOF haptic device and Yuta Itoh from FAR for inspiring discussions while developing the reference orientation algorithm.

\section{REFERENCES}

[1] P. T. Boggs and J. E. Rogers. Orthogonal Distance Regression. Contemporary Mathematics, 112:183-194, 1990.

[2] S. M. B. I. Botden, S. N. Buzink, M. P. Schijven, and J. J. Jakimowicz. Augmented versus Virtual Reality Laparoscopic Simulation: What Is the Difference? World Journal of Surgery, 31(4):764-772, 2007.

[3] M. C. Çavuşoğlu, D. Feygin, and F. Tendick. A Critical Study of the Mechanical and Electrical Properties of the Phantom Haptic Interface and Improvements for High Performance Control. Presence: Teleoperators and Virtual Environments, 11(6):555-568, 2002.

[4] F. I. Cosco, C. Garre, M. Muzzupappa, and M. A. Otaduy. Augmented Touch without Visual Obtrusion. In Proc. of the 8th IEEE International Symposium on Mixed and Augmented Reality, pages 99-102, Orlando, FL, USA, 2009.

[5] M. Csongei, L. Hoang, U. Eck, and C. Sandor. ClonAR: Rapid redesign of realworld objects. In Proc. of the 11th IEEE International Symposium on Mixed and Augmented Reality, pages 277-278, Atlanta, GA, USA, 2012.

[6] M. J. Fu, A. D. Hershberger, K. Sano, and M. C. Cavusoglu. Effect of VisuoHaptic Co-location on 3D Fitts' Task Performance. In Proc. of the International Conference on Intelligent Robots and Systems (IROS), 2011 IEEE/RSJ, pages 3460-3467, San Francisco, CA, USA, 2011.

[7] M. Harders and G. Szekely. Calibration, Registration, and Synchronization for High Precision Augmented Reality Haptics. IEEE Transactions on Visualization and Computer Graphics, 15(1):138-149, 2009.

[8] J. M. Hollerbach and C. W. Wampler. The Calibration Index and Taxonomy for Robot Kinematic Calibration Methods. The International Journal of Robotics Research, 15(6):573-591, Dec. 1996.

[9] B. K. P. Horn. Closed-form Solution of Absolute Orientation using Unit Quaternions. Journal of the Optical Society of America, 4(4):629-642, 1987.

[10] M. Huber, S. Michael, and G. Klinker. Temporal Calibration in Multisensor Tracking Setups. 9. Workshop der GI-Fachgruppe Virtuelle und Erweiterte Realität (VR-AR 2012), pages 201-212, Sept. 2012.

[11] M. Ikits, C. D. Hansen, and C. R. Johnson. A Comprehensive Calibration and Registration Procedure for the Visual Haptic Workbench. In Proc. of the 9th Eurographics Workshop on Virtual Environments, pages 247-254, New York, NY, USA, May 2003.

[12] B. Knoerlein and M. Harders. Comparison of tracker-based to tracker-less haptic device calibration. In World Haptics Conference (WHC), pages 119-124, Istanbul, Turkey, 2011.

[13] T. H. Massie and J. K. Salisbury. The Phantom Haptic Interface: A Device for Probing Virtual Objects. In Proc. of the ASME Winter Annual Meeting, Symposium on Haptic Interfaces for Virtual Environment and Teleoperator Systems, pages 295-300, Chicago, IL, USA, Jan. 1994.

[14] B. W. Mooring, Z. S. Roth, and M. R. Driels. Fundamentals of Manipulator Calibration. John Wiley \& Sons, Inc., 1991.

[15] D. Pustka, M. Huber, M. Bauer, and G. Klinker. Spatial Relationship Patterns: Elements of Reusable Tracking and Calibration Systems. In Proc. of the 5th International Symposium for Mixed and Augmented Reality, pages 88-97, Santa Barbara, CA, USA, 2006.

[16] D. Pustka and G. Klinker. A System Architecture for Ubiquitous Tracking Environments. In Proc. of the 6th IEEE and ACM International Symposium on Mixed and Augmented Reality, pages 1-4, Nara, Japan, 2007.

[17] P. Rhienmora, K. Gajananan, P. Haddawy, M. N. Dailey, and S. Suebnukarn. Augmented Reality Haptics System for Dental Surgical Skills Training. In Proc. of the 17th ACM Symposium on Virtual Reality Software and Technology, pages 97-98, Hong Kong, 2010.

[18] C. Sandor and S. Uchiyama. Exploring Visuo-Haptic Mixed Reality. Technical Report PRMU 106, Tokyo, Japan, 2007.

[19] R. Y. Tsai and R. K. Lenz. A New Technique for Fully Autonomous and Efficient 3D Robotics Hand/Eye Calibration. IEEE Transactions on Robotics and Automation, 5(3):345-358, June 1989.

[20] M. Tuceryan, D. S. Greer, and R. T. Whitaker. Calibration Requirements and Procedures for a Monitor-Based Augmented Reality System. IEEE Transactions on Visualization and Computer Graphics, 1(3):255-273, 1995.

[21] J. Vallino and C. Brown. Haptics in Augmented Reality. In Proc. of the IEEE International Conference on Multimedia Computing and Systems, pages 195200, 1999.

[22] D. Wang, Y. Zhang, W. Zhou, H. Zhao, and Z. Chen. Collocation Accuracy of Visuo-Haptic System: Metrics and Calibration. IEEE Transactions on Haptics, 4(4):321-326, 2011. 